\title{
Schwannoma esofágico gigante, un diagnóstico de exclusión: reporte de caso
}

\section{Giant esophageal schwannoma, a diagnosis by exclusion: Case report}

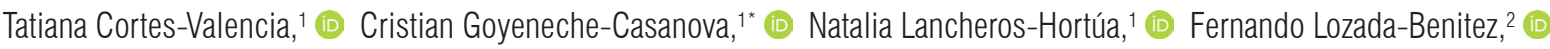 \\ Diana Fierro-Rodriguez. ${ }^{3}$
}

\begin{abstract}
Gacceso abierto
Citación:

Cortes-Valencia T, Goyeneche-Casanova C, Lancheros-Hortúa N, Lozada-Benitez F, FierroRodriguez D. Schwannoma esofágico gigante, un diagnóstico de exclusión: reporte de caso. Rev Colomb Gastroenterol. 2021;36(Supl.1):37-41. https://doi.org/10.22516/25007440.516

Médico general, Fundación Universitaria de Ciencias de la Salud. Bogotá, Colombia. 2 Residente de tercer año, Radiología e imágenes diagnósticas, Fundación Universitaria de Ciencias de la Salud. Bogotá, Colombia. ${ }^{3}$ Radióloga, instructora asistente, Fundación Universitaria de Ciencias de la Salud. Bogotá, Colombia.
\end{abstract}

*Correspondencia: Cristian Goyeneche-Casanova cygoyeneche@fucsalud.edu.co

Fecha recibido: $28 / 02 / 20$ Fecha aceptado: 06/09/20

\section{Resumen}

Introducción: Los schwannomas esofágicos son tumores de elementos perineurales de la vaina nerviosa de la célula de Schwann en los nervios periféricos y representan el $2 \%$ de los tumores primarios de esófago. Su difícil diagnóstico está dado por la baja incidencia que este presenta; sin embargo, es una etiología que debe considerarse debido a que su comportamiento clínico e imagenológico es rápidamente progresivo e inusual, comparado con otros tumores benignos de esófago. Caso clínico: paciente femenina de 38 años con cuadro clínico de disfagia de 1 año de evolución, con endoscopia de vías digestivas altas y tomografía axial computarizada (TAC) de tórax contrastada que evidencian una masa a nivel del esófago cervical y torácico transmural, que obstruye la luz y ejerce el efecto de masa sobre la tráquea. Se realizó una biopsia que reportó un tumor fusocelular con inmunohistoquímica positiva para el marcador S100, por lo que se diagnosticó un schwannoma esofágico. En la actualidad, la paciente se encuentra en controles periódicos y en espera del concepto por oncología clínica para proponer tratamiento no quirúrgico dado el compromiso adyacente. Conclusión: Ante la sospecha de tumor primario de esófago, la primera impresión diagnóstica según la incidencia será un leiomioma, pero con nuestro reporte de caso de schwannoma esofágico, se resalta que, en un cuadro clínico no usual, este es un diagnóstico diferencial que requiere atención precoz para evitar complicaciones y secuelas en los pacientes.

\section{Palabras clave (DeCS)}

Schwannoma, esófago, neurilemoma, tumor primario de esófago, reporte de caso.

\begin{abstract}
Introduction: Esophageal schwannomas are tumors of the perineural components of the Schwann cell nerve sheath in peripheral nerves and account for $2 \%$ of primary esophageal tumors. Its low incidence makes diagnosis challenging; however, this etiology should be considered because its clinical and imaging behavior is rapidly progressive and unusual compared to other benign esophageal tumors. Case study: A 38-year-old female patient with a 1-year history of dysphagia underwent upper digestive tract endoscopy and contrast chest CT showing a mass at the cervical and transmural thoracic esophagus level, obstructing the lumen and exerting a mass effect on the trachea. A biopsy revealed a spindle cell tumor with positive immunohistochemistry for the S100 marker, leading to the diagnosis of esophageal Schwannoma. The patient is currently undergoing regular check-ups and is awaiting the advice of a clinical oncologist to recommend non-surgical treatment options due to the involvement of adjacent structures. Conclusion: The first diagnostic impression in the case of a suspected primary esophageal tumor would be a leiomyoma based on its incidence. The present case report of an esophageal schwannoma emphasizes that this is a differential diagnosis that requires timely treatment to avoid complications and sequelae in patients.
\end{abstract}

Keywords (DeCS)

Schwannoma; Esophagus; Neurilemoma; Primary esophageal tumor; Case report. 


\section{INTRODUCCIÓN}

Los schwannomas (neurilemomas) esofágicos son tumores que se desarrollan de elementos perineurales de la vaina nerviosa de la célula de Schwann en los nervios periféricos (1), tienen una incidencia poco frecuente y son de carácter benigno en la mayoría de casos. Se caracterizan histológicamente por nódulos linfoides periféricos, atipia nuclear benigna y células en forma de huso (2-4).

Los schwannomas esofágicos representan 1 de cada 5000 pacientes con masas a este nivel. Su mayor frecuencia de presentación está entre la tercera y séptima década de la vida, con una relación hombre/mujer de 1:3 (5). El 5 \% de los casos diagnosticados se puede asociar con neurofibromatosis tipo I, que son los de pronóstico más agresivo y presentación temprana (6). Clínicamente, el schwannoma esofágico puede cursar de forma asintomática; sin embargo, en la mayoría de casos se diagnostica en una fase avanzada, y se manifiesta principalmente con síntomas como disfagia de moderada a grave y disnea, asociados con dolor torácico, epigastralgia, tos, hemoptisis, palpitaciones y neumonía, dependiendo de su ubicación y el efecto de masa (6).

Para una aproximación diagnóstica, los estudios por imágenes (radiografía $[\mathrm{RX}]$, tomografía axial computarizada $[\mathrm{TAC}]$, resonancia magnética nuclear $[\mathrm{RMN}])$ brindan mayor información sobre las características anatómicas del tumor. Estos hallazgos no permiten distinguir entre un schwannoma y otros tumores de la submucosa, debido a que comparten características imagenológicas similares
(7). El diagnóstico correcto recae en el estudio histopatológico e inmunohistoquímico, y el marcador tumoral S-100 es el más específico y sensible para esta patología, dado que en la totalidad de los reportes de casos en la literatura para schwannoma resulta positivo, comparado con otros marcadores utilizados $(6,8)$.

\section{CASO CLÍNICO}

Se presenta el caso de una paciente femenina de 38 años con cuadro clínico consistente en disfagia de 1 año de evolución asociado con episodios ocasionales de hematemesis, por lo que se realizó una endoscopia de vías digestivas altas que evidenció una lesión endoluminal, friable e irregular que se localizó a $27 \mathrm{~cm}$ desde la arcada dentaria y de $12 \mathrm{~cm}$ de longitud, de la que se tomó una muestra para estudio histopatológico, que reportó una lesión benigna compatible con leiomioma. Sin embargo, se amplió el estudio con una TAC de tórax contrastada en la que se observó una masa tumoral a nivel del esófago cervical de $128 \mathrm{~mm}$ x 66 mm x 84 en diámetros $\mathrm{L} \times \mathrm{AP} \times \mathrm{T}$, que se extiende hasta el esófago torácico transmural, exofítica, con realce homogéneo dependiente de la pared anterior la cual condiciona la obstrucción de la luz y ejerce un efecto de masa sobre la tráquea en un $70 \%$ aproximadamente (Figura 1A y B).

La paciente fue llevada a junta medicoquirúrgica por inquietudes acerca de los hallazgos histopatológicos, por lo que decidió llevar a biopsia nuevamente, esta vez por toracoscopia de la lesión mediastinal descrita que reportó un
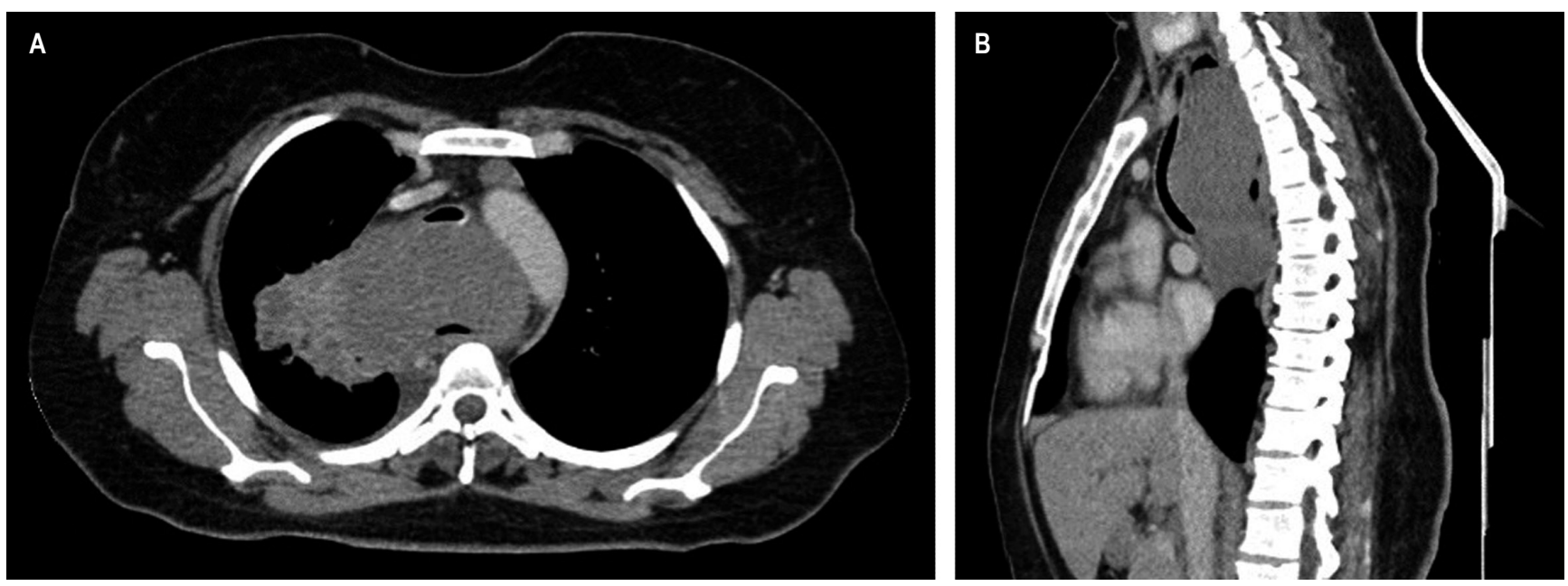

Figura 1. TAC de tórax contrastado. Masa en el mediastino medio de 128 x 66 x $84 \mathrm{~mm}$ en diámetros L x AP x T, respectivamente, sin realce significativo posterior a la administración de medio de contraste, homogénea, de contornos parcialmente definidos lobulados, con extensión desde C7 hasta T7 con un efecto compresivo sobre las estructuras mediastinales adyacentes, en especial con la tráquea que desplaza el tejido en sentido anterior con disminución de su calibre sobre un 73 \% aproximadamente; y en el esófago, donde se aprecia parcialmente su luz. Ganglios linfáticos subcentimétricos de localización prevascular, el de mayor tamaño con eje corto de $8 \mathrm{~mm}$; a nivel paraaórtico se observan ganglios linfáticos con eje corto de $10 \mathrm{~mm}$. A. Corte axial. B. Reconstrucción sagital. 
tumor fusocelular con inmunoperfil que favorece el tumor de vaina nerviosa periférica sin criterios claros de atipia celular (Figura 2) e inmunohistoquímica con positividad para proteína $S 100$ y negatividad para leucemia mielógena aguda (LMA), H-Caldesmon, desmina, CD117, DOG-1, CD34, CKAE1/AE3 y STAT-6. El índice de proliferación celular (Ki-67) es del $5 \%$. Se concluyó que la masa corresponde a un schwannoma esofágico. Se realizó nueva TAC de tórax con contraste en la que se evidenció un aumento del tamaño de la masa con compromiso de la pleura mediastinal y el parénquima pulmonar apical derecho, en contacto con la arteria pulmonar derecha, el cayado aórtico y el cayado de la vena ácigos. Se presentó en junta quirúrgica nuevamente, con los servicios de cirugía de tórax, oncológica y esofágica, y se consideró que debido a la extensión, tamaño tumoral y compromiso vascular, el tumor se hace irresecable quirúrgicamente. Se derivó a oncología clínica para considerar otra opción terapéutica con el objetivo de aminorar los síntomas. Adicional al cuadro clínico descrito durante el transcurso de la patología, la paciente presentó episodios de neumonía que requirieron un manejo antibiótico y soporte ventilatorio de forma intrahospitalaria (Figura 3).

\section{DISCUSIÓN}

Los tumores primarios de esófago representan el $2 \%$ de los casos; de estos, el $80 \%$ son leiomiomas y solo el $1 \%$ son schwannomas; el porcentaje restante corresponde a
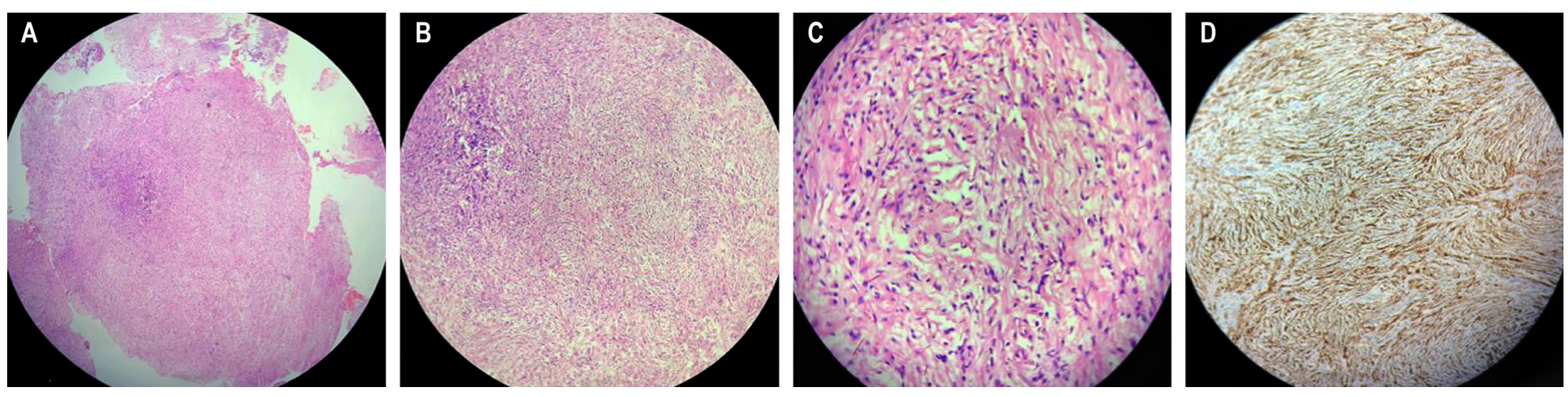

Figura 2. Estudio microscópico. Lesión neoplásica constituida por células fusiformes y algunas pleomórficas sobre un estroma fibrocolágeno asociado con la presencia de infiltrado inflamatorio linfocítico. A. Tinción con hematoxilina-eosina (HE; aumento x 4). B. Tinción con HE (aumento x 10). C. Tinción con HE (aumento x 40). D. Inmunohistoquímica para proteína S100 positivo.
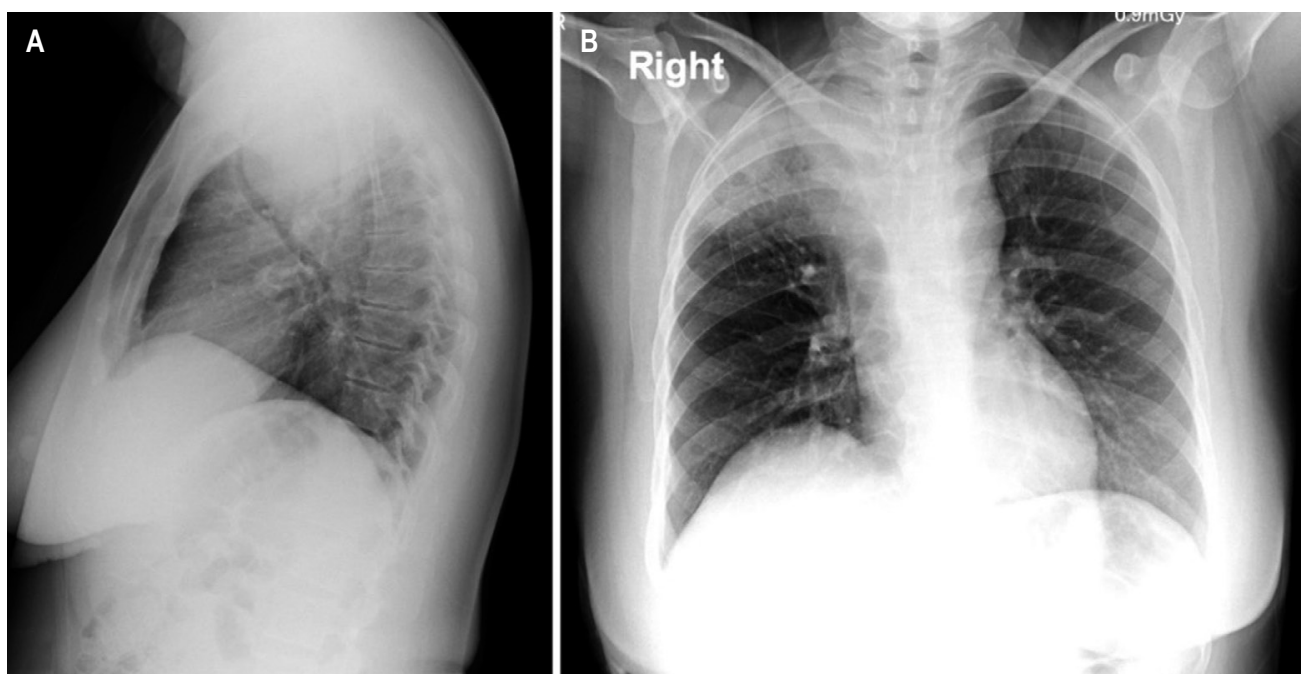

Figura 3. RX de tórax. Masa en el mediastino medio, con un componente aéreo interno en su aspecto superior que no descarta cavitación; esta imagen se comunica con consolidación adyacente del lóbulo superior derecho, lo cual no descarta un trayecto fistuloso. La masa genera un importante efecto compresivo sobre las estructuras adyacentes, especialmente sobre la tráquea, la cual disminuye su calibre en un $73 \%$ aproximadamente. Masa con compromiso de la pleura mediastinal y el parénquima pulmonar apical derecho en contacto con la arteria pulmonar derecha, cayado aórtico y cayado de la vena ácigos sin signos de infiltración. A. Proyección lateral. B. Proyección posteroanterior. 
otros tipos histopatológicos $(2,8,9)$. Los schwannomas esofágicos son poco frecuentes y de carácter benigno en su mayoría. En la literatura se han descrito 54 casos hasta el momento, 6 de ellos malignos, y al comparar estos reportes se encontró que la paciente de este caso está en el rango de edad entre los 30 a 60 años (6), pero con características histológicas indeterminadas para clasificar el tumor como benigno o maligno, ya que bajo el estudio microscópico se encontraron componentes de células fusiformes y algunas pleomórficas sin criterios claros de atipia sobre un estroma fibrocolágeno asociado con la presencia de infiltrado inflamatorio linfocítico, lo que podría sugerir preliminarmente una masa benigna y, en pruebas de inmunohistoquímica, la proteína S100 positiva como estándar de oro al momento del diagnóstico de schwannoma, aunque con un índice de proliferación celular (Ki-67) del $5 \%$, que es mayor al promedio reportado en la literatura (2\%-3\%) (10). De lo anterior se podría deducir un desarrollo inusual de progresión rápida, teniendo en cuenta que diversos autores proponen malignidad cuando existe una masa mayor a $60 \mathrm{~mm}$ en uno de sus ejes (6). Las imágenes diagnósticas reportadas describieron la masa en $66 \mathrm{~mm}$ en su eje más corto, lo que orienta hacia la malignidad en este caso.

En la literatura es común encontrar pacientes con historia de masas que sean diagnosticadas en el momento inicial como leiomiomas, pero si su evolución es inusual puede estar indicada nuevamente la toma de biopsia mediante otro tipo de técnica ante la sospecha de schwannoma (4, $8,11)$. Este abordaje diagnóstico se debe a que la mayoría de las biopsias son tomadas de forma superficial por endoscopia, por lo que se encuentra principalmente tejido submucoso sin obtener componentes de la vaina nerviosa periférica (12). En este tipo de tumor es importante la técnica de obtención de la muestra para el estudio patológico e inmunohistoquímico. En nuestro caso, se obtuvo por medio de toracoscopia, pero el enfoque inicial fue similar a lo descrito en la literatura, pues se realizó un diagnóstico preliminar de leiomioma; después de un análisis interdisciplinar, se solicitó una nueva biopsia para el diagnóstico de schwannoma. Por esta razón, se podría explicar la causa de subregistro de esta patología.

En los estudios por tomografía, los schwannomas comparten características con otros tumores esofágicos, pero se describen como imágenes redondeadas u ovaladas con bordes definidos, atenuación igual o menor que los tejidos blandos, realce al medio de contraste homogéneo o heterogéneo (dependiendo de la presencia de contenido lipídico en las células de Schwann), tejido adiposo perineural atra- pado y espacios quísticos o áreas calcificadas pequeñas, las cuales se encuentran en el $5 \%$ a $10 \%$ de los casos (13). Los hallazgos de la paciente son similares a los descritos anteriormente, pero la extensión a estructuras adyacentes como la tráquea (obliteración del $70 \%$ ); esófago con leve apreciación de su luz y efecto de masa sobre el cayado aórtico, la arteria pulmonar derecha y el cayado de la vena ácigos pueden condicionan el abordaje.

Los schwannomas esofágicos no son radio- ni quimiosensibles, pero pueden ser resecados por medio de técnicas quirúrgicas como toracotomía posterolateral derecha o izquierda, cirugía toracoscópica videoasistida y cirugía torácica videoasistida por robot adicionada a una enucleación o esofagectomía (5), dependiendo del tamaño tumoral, del compromiso del esófago y otras estructuras adyacentes; inclusive en los casos reportados de schwannoma maligno el tratamiento fue quirúrgico con resultados favorables y sin evidencia de recidiva (12). Para nuestro caso, la cirugía fue descartada dado el compromiso de diferentes estructuras mediastinales y el tamaño del tumor. El abordaje quirúrgico del leiomioma o del schwannoma son similares (9). Esta particularidad puede llevar a inferir que, asociado con el subregistro por mala obtención de muestras y la resección tumoral bajo un mismo esquema quirúrgico, facilitaría ese mismo subregistro.

Actualmente, la paciente continúa asistiendo a controles periódicos, con los que se busca vigilar el compromiso de su funcionalidad a causa del efecto de masa que ejerce el tumor sobre el esófago y la tráquea, y en espera del concepto por oncología clínica para evaluar alternativas de manejo.

\section{CONCLUSIÓN}

Se reporta un caso de schwannoma esofágico con una presentación clínica inicial no usual, con positividad para el marcador $\mathrm{S} 100$ y un Ki-67 mayor a lo reportado en la literatura; sin embargo, la paciente no presentó características radiológicas ni anatomopatológicas concluyentes para definir malignidad, por lo que el tratamiento resultó ser limitado, llevando a descartar el abordaje quirúrgico, que continúa siendo la opción terapéutica más acertada.

Los leiomiomas son el tumor primario de esófago más frecuente, pero es importante obtener la biopsia por la técnica adecuada y la evaluación anatomopatológica e inmunohistoquímica que especifique con certeza el tipo de histología asociada con la clínica y la evolución radiológica, pero ante una clínica no usual, un diagnóstico diferencial en los tumores primarios es el schwannoma de esófago. 


\section{REFERENCIAS}

1. Brunicardi FC, Billiar TR, Dunn DL, Hunter JG, Matthews JB, Pollock RE, Wu JX. Schwartz. Principios de cirugía. Autoevaluación y repaso. 10. a edición. Mc-Graw Hill; 2010.

2. Kitada M, Matsuda Y, Hayashi S, Ishibashi K, Oikawa K, Miyokawa N. Esophageal schwannoma: a case report. World J Surg Oncol. 2013;11:253. https://doi.org/10.1186/1477-7819-11-253

3. Zhu L, Li W, Zhu Z, Chai Y. Benign esophageal schwannoma: A case report and review of literature. Niger J Clin Pract. 2019;22(5):731-733. https://doi.org/10.4103/njcp.njcp_142_18

4. Kobayashi N, Kikuchi S, Shimao H, Hiki Y, Kakita A, Mitomi H, Ohbu M. Benign esophageal schwannoma: report of a case. Surg Today. 2000;30(6):526-9. https://doi.org/10.1007/s005950070120

5. Morales-Maza J, Pastor-Sifuentes FU, Sánchez-Morales GE, Ramos ES, Santes O, Clemente-Gutiérrez U, PimientaIbarra AS, Medina-Franco H. Clinical characteristics and surgical treatment of schwannomas of the esophagus and stomach: A case series and systematic review. World J Gastrointest Oncol. 2019;11(9):750-760. https://doi.org/10.4251/wjgo.v11.i9.750

6. Souza LCA, Pinto TDA, Cavalcanti HOF, Rezende AR, Nicoletti ALA, Leão CM, Cunha VC. Esophageal schwannoma: Case report and epidemiological, clinical, surgical and immunopathological analysis. Int J Surg Case Rep. 2019;55:69-75. https://doi.org/10.1016/j.ijscr.2018.10.084

7. Souza LCA, Pinto TDA, Cavalcanti HOF, Rezende AR, Nicoletti ALA, Leão CM, Cunha VC. Esophageal schwan- noma: Case report and epidemiological, clinical, surgical and immunopathological analysis. Int J Surg Case Rep. 2019;55:69-75. https://doi.org/10.1016/j.ijscr.2018.10.084

8. Zhang Y, Han Y, Xiang J, Li H. Robot-assisted enucleation of large dumbbell-shaped esophageal schwannoma: a case report. BMC Surg. 2018;18(1):36. https://doi.org/10.1186/s12893-018-0370-y

9. Quintero Rivera JC, Arias Morales Y, Carcacía Hermilla I, Prieto Casal PL, Armesto Fernandez MJ, Ourense ES. Schwannomas extracraneales. Hallazgos radiológicos y diagnóstico diferencial. Congreso Nacional de la Sociedad de Radiología Española 2012. Granada; 2012.

10. Park BJ, Carrasquillo J, Bains MS, Flores RM. Giant benign esophageal schwannoma requiring esophagectomy. Ann Thorac Surg. 2006;82(1):340-2. https://doi.org/10.1016/j.athoracsur.2005.09.042

11. Wang S, Zheng J, Ruan Z, Huang H, Yang Z, Zheng J. Long-term survival in a rare case of malignant esophageal schwannoma cured by surgical excision. Ann Thorac Surg. 2011;92(1):357-8. https://doi.org/10.1016/j.athoracsur.2011.01.045

12. Mishra B, Madhusudhan KS, Kilambi R, Das P, Pal S, Srivastava DN. Malignant Schwannoma of the Esophagus: A Rare Case Report. Korean J Thorac Cardiovasc Surg. 2016;49(1):63-6. https://doi.org/10.5090/kjtcs.2016.49.1.63

13. Walker C, Chung J. Muller's Imaging of the Chest. 2. ${ }^{\text {a }}$ edición. Elsevier; 2018. 